\title{
EXAMPLES RELATING TO NORMALITY IN TOPOLOGICAL SPACES
}

\author{
BY \\ H. H. CORSON
}

1. Introduction. In a recent paper [3], Mansfield has studied various topological properties which lie between normality and paracompactness. He raised several questions concerning equivalence among these properties. Some of these questions are answered here by proving that the $\Sigma$-products which I recently studied in detail furnish counterexamples. I wish to thank Melvin Henriksen for his suggestion that the $\Sigma$-products might serve the purpose to which they are put in these theorems.

Those who are interested in the theory of $\Sigma$-products will notice that these results sharpen the known facts, although at the expense of techniques which are different and more involved. Hence, for simplicity's sake, only $\Sigma$ products of copies of the integers will be presented. These methods can be used to prove analogous theorems where each of the factors is separable metric and complete; but what is true beyond this is open, except for the results in [2].

2. Notation and statement of the main theorem. If for each $\alpha \in A$ there is given a copy of the integers $J_{\alpha}$, then the $\Sigma$-product of the $J_{\alpha}$ is defined to be the set of points $p$ in the topological product $P$ of the $J_{\alpha}$ such that $p_{\alpha} \neq 0$ for at most a countable number of coordinates. $\Sigma$ is given the topology it inherits from $P$.

The properties studied by Mansfield are modifications of the concept of full normality which was introduced by Tukey. Let us define this term first. A topological space $X$ is fully normal if for each open cover $\mathcal{U}$ of $X$ there is an open refinement $\mathcal{U}$ of $\mathcal{U}$ such that, given any point $x \in X$ and any subcollection $v_{x} \subset v$ with $x \in \cap v_{x}$, there is some $U \in \mathcal{u}$ with $U v_{x} \subset U$. Now, if $\mathfrak{m}$ is a cardinal number, $m$-fully normal is defined similarly with the alteration that $v_{x}$ has cardinality less than or equal to $\mathrm{m}$. Also, almost $\mathrm{m}$-fully normal is defined by altering the definition of fully normal to require only that for each subset $M$ of cardinality $m$ of each $U v_{x}$ there is a $U \in \mathcal{U}$ such that $M \subset U$. Note that these properties are inherited by closed subspaces $[3$, Theorem 4.1].

By the references or theorems in Mansfield's paper one will see that the following sequence of implications holds for all infinite $\mathrm{m}$. (Some remarks on the case where $\mathfrak{m}$ is finite are in the last section.)

This paper has been submitted to and accepted for publication by the Proceedings of the American Mathematical Society. It has been transferred to these Transactions, with the consent of the author, for technical reasons. Received by the editors August 8, 1960. 
paracompact $\Leftrightarrow$ fully normal $\Rightarrow \mathfrak{m}$-fully normal $\Rightarrow$ almost $\mathfrak{m}$-fully normal $\Rightarrow$ countably paracompact and normal.

(Recall that $X$ is paracompact if each open cover has an open, locally finite refinement. Also, $X$ is countably paracompact if each countable open cover has an open, locally finite refinement.) Moreover, these are the only valid implications among these properties, since the following theorem settles the only case left open by Mansfield.

TheOREM 1. Let $\Sigma$ be the $\Sigma$-product of uncountably many copies of the integers. Then $\Sigma$ is almost $\aleph_{0}$-fully normal, but not $\aleph_{0}$-fully normal.

3. Proof of the positive part of Theorem 1. Before embarking on this rather involved proof, the reader may appreciate an example of a simple cover for $\Sigma$-yet a cover which is very much like the refinement that will be constructed in this section. For each $\alpha \in A$, let

$$
W(\alpha)=\left\{x \in \Sigma: x_{\alpha}=0\right\} .
$$

Then $w=\{W(\alpha): \alpha \in A\}$ is the cover to which I refer. It is easy to see that any countable subset of $\Sigma$ is contained in an appropriate $W(\alpha)$. The general idea of the following proof is to construct a refinement as much like $W$ as possible. Furthermore, $W$ is also used in the next section to prove that $\Sigma$ is not $\aleph_{0}$-fully normal.

From the proof that $\Sigma$ is almost $\boldsymbol{\aleph}_{0}$-fully normal it is possible to isolate that part that is similar to those techniques developed in [2], and this is presented in the proof to Lemma 1 . Note that a basic open set $U$ is an open cylinder in $\Sigma$ such that $U_{\alpha}$ consists of one point in $J_{\alpha}$ for the $\alpha$ where $U_{\alpha} \neq J_{\alpha}$.

LEMMA 1. For each open cover $u$ of $\Sigma$ by basic open sets, there is a countable subset $K \subset A$ and a subcover $\mathcal{V}$ of $U$ such that $V_{\alpha}=0$ or $J_{\alpha}$ for each $V \in \mathcal{V}$ and $\alpha \in^{\prime} K$.

Proof. Suppose the lemma is false. Let $K_{1}$ be a countable subset of $A$, and arrange $K_{1}$ in a simple sequence. Denote by $\alpha_{1}$ the first member of $K_{1}$. Let $u_{1}$ be the set of $U \in \mathcal{u}$ such that $U_{\alpha}=0$ or $J_{\alpha}$ if $\alpha \in^{\prime} K_{1}$. For $n$ an integer, define

$$
\Sigma\left(\alpha_{1}, n\right)=\left\{x \in \Sigma: x_{\alpha_{1}}=n\right\} \text {. }
$$

Since there are only countably many $\Sigma\left(\alpha_{1}, n\right)$, and since we have assumed that the lemma is false, there exists an $n_{1}$ such that $\Sigma\left(\alpha_{1}, n_{1}\right)$ has this property: For each cover $w$ of $\Sigma\left(\alpha_{1}, n_{1}\right)$ by elements of $\mathcal{u}$ and each countable $K \subset A$, there is a $W \in W$ such that $W_{\alpha} \neq 0$ or $J_{\alpha}$ for at least one $\alpha \in \in^{\prime} K$. In particular one may choose $x_{1} \in \Sigma\left(\alpha_{1}, n_{1}\right)$ such that $x_{1} \in \in^{\prime} \cup u_{1}$.

For the next step of the induction, define

$$
K_{2}=\left\{\alpha \in A:\left(x_{1}\right)_{\alpha} \neq 0\right\} \text {, }
$$


and arrange $K_{2}$ in a simple sequence. Suppose that $r_{1}, r_{2}, r_{3}, r_{4}$ are integers, and assume that $\alpha_{1}, \alpha_{2}$ are the first two elements of $K_{1}$ while $\alpha_{3}, \alpha_{4}$ are the first two elements of $K_{2}$. Define

$$
\Sigma\left(\alpha_{1} \alpha_{2} \alpha_{3} \alpha_{4}, r_{1} r_{2} r_{3} r_{4}\right)=\left\{x \in \Sigma: x_{\alpha_{i}}=r_{i}, i=1, \cdots, 4\right\} .
$$

Let

$$
\mathfrak{u}_{2}=\left\{U \in \mathcal{u}: U_{\alpha}=0 \text { or } J_{\alpha} \text { if } \alpha \in^{\prime} K_{1} \cup K_{2}\right\} \text {. }
$$

Since there are only countably many $\Sigma\left(\alpha_{1} \cdots \alpha_{4}, r_{1} \cdots r_{4}\right)$, since the lemma is assumed to be false, and since $n_{1}$ was chosen as above, there are $n_{2}, n_{3}, n_{4}$ with the property: For each cover $W$ of $\Sigma\left(\alpha_{1} \alpha_{2} \alpha_{3} \alpha_{4}, n_{1} n_{2} n_{3} n_{4}\right)$ by elements of $u$ and each countable $K \subset A$, there is a $W \in W$ such that $W_{\alpha} \neq 0$ or $J_{\alpha}$ for some $\alpha \in^{\prime} K$. In particular one may choose $x_{2} \in \Sigma\left(\alpha_{1} \cdots \alpha_{4}, n_{1} \cdots n_{4}\right)$ such that $x_{2} \in^{\prime} \cup u_{2}$.

Continue this process, taking the first $s$ members of each $K_{i}, i \leqq s$, at the $s$ th step. One can prove that $x_{i}$ converges to some $x_{0} \in \Sigma$ as in [2, Theorem 1]. Choose a $U \in \mathcal{u}$ such that $x_{0} \in U$. Let $N$ be an integer such that $x_{i} \in U$ if $i \geqq N$. Let $M$ be an integer such that $U_{\alpha}=J_{\alpha}$ if

$$
\alpha \in U\left\{K_{i}: i=M+1, M+2, \cdots\right\},
$$

but $\alpha \in \in^{\prime} K_{1} \cup \ldots \cup K_{M}$. Then define $T$ to be $\max (M, N)$. If $\mathfrak{u}_{T}$ is the subset of $\mathcal{U}$ defined at the $T$ th stage of the induction, one sees that $U \in \mathcal{U}_{T}$ for the following reason: If $U_{\alpha} \neq 0$ or $J_{\alpha}$, then $\alpha \in K_{1} \cup \ldots \cup K_{M}$ because $x_{0} \in U$ on the one hand, and $M$ was chosen as it was. This gives a contradiction since $x_{T} \in U \in \mathcal{u}_{T}$, and $x_{T}$ was not supposed to be in $U \mathfrak{u}_{T}$.

Having proved Lemma 1, we now note that in order to prove Theorem 1, it suffices to show that every cover $u$ by basic open sets has a refinement of the required type. Let $V$ and $K$ be the sets in the statement of Lemma 1. Arrange $K$ in a simple sequence, and let $\alpha_{1} \cdots \alpha_{s}$ be the first $s$ elements of $K$. Let $n_{1} \cdots n_{s}$ be integers. For each $V \in V$ such that $n_{i} \in V_{\alpha_{i}}, i=1 \cdots s$, and such that $V_{\alpha}=J_{\alpha}$ for $\alpha \in K \backslash\left\{\alpha_{1} \cdots \alpha_{\varepsilon}\right\}$, define $V^{\prime}$ to be the basic open set such that

$$
\left(V^{\prime}\right)_{\alpha_{i}}=n_{i}, \quad i=1 \cdots s
$$

and

$$
\left(V^{\prime}\right)_{\alpha}=V_{\alpha}, \quad \alpha \neq \alpha_{i}, \quad i=1 \cdots s .
$$

Denote by $g_{s}$ the set of all such $V^{\prime}$ for $V \in V$ and integers $n_{1} \cdots n_{s}$.

The following assertion will now be proved: Let $\mathcal{G}=U_{1}^{\infty} \mathcal{G}_{\text {, }}$ and let $\Sigma_{K}$ $=\left\{x \in \Sigma: x_{\alpha}=0\right.$ if $\left.\alpha \in \in^{\prime} K\right\}$. Then for $x \in \Sigma_{K}$ and each countable $K^{\prime} \supset K$ there is a $U \in \mathcal{S}$ such that $x \in U$ and $U_{\alpha}=J_{\alpha}$ if $\alpha \in K^{\prime} \backslash K$. 
For suppose the assertion were false for some $x \in \Sigma_{K}$ and some $K^{\prime}$. Let $x^{\prime} \in \Sigma$ be defined by

$$
\left(x^{\prime}\right)_{\alpha}= \begin{cases}x_{\alpha}, & \text { if } \alpha \in K, \\ 1, & \text { if } \alpha \in K^{\prime} \backslash K, \\ 0, & \text { otherwise. }\end{cases}
$$

Since any $U \in \mathcal{G}$ with $x \in U$ has the property that $U_{\alpha} \neq J_{\alpha}$ for some $\alpha \in K^{\prime} \backslash K$, and since $\mathcal{G}$ has the relation to $V$ that was described above, $U_{\alpha}=0$ for some $\alpha \in K^{\prime} \backslash K$. Hence $x^{\prime} \in$ Ug. However, this is a contradiction, since it is easy to see that $\mathcal{G}$ is a cover for $\Sigma$ because $v$ is a cover.

Let $\Omega$ be the first uncountable ordinal. For $x \in \Sigma_{K}$ and $\beta<\Omega$, define countable sets $K_{\beta}(x) \subset A$ and $U_{\beta}(x) \in \mathcal{G}$ inductively so that they have these properties: First, $K \subset K_{\beta}(x) K_{\delta}(x)$ if $\beta<\delta$. Second, $x \in U_{\beta}(x)$ and $\left(U_{\beta}(x)\right)_{\alpha}=J_{\alpha}$ if $\alpha \in K_{\beta}(x) \backslash K$. Finally, if $\left(U_{\delta}(x)\right)_{\alpha} \neq J_{\alpha}$ for $\delta<\beta$, then $\alpha \in K_{\beta}(x)$. Since, for $x \in \Sigma_{K}$, there are $\aleph_{1}$ of the $U_{\beta}(x)$, it follows that there is a subset $\left\{\alpha_{1} \cdots \alpha_{8}\right\}$ $C K$ and integers $n_{1} \cdots n_{s}$ (depending on $x$ ) with the property: For uncountably many $U_{\beta}(x), \beta<\Omega$, one has

$$
\left(U_{\beta}(x)\right)_{\alpha_{i}}=n_{i}, \quad i=1 \cdots s,
$$

and

$$
\left(U_{\beta}(x)\right)_{\alpha}=J_{\alpha}, \quad \text { for } \alpha \in K \backslash\left\{\alpha_{1} \cdots \alpha_{s}\right\} .
$$

Denote this uncountable subset of $\mathcal{G}$ by $\mathfrak{F C}(x)$, where $x \in \Sigma_{K}$.

First, $\mathfrak{F C}=U\left\{\mathfrak{F C}(x): x \in \Sigma_{K}\right\}$ is a cover for $\Sigma$. To show this, let $y \in \Sigma$ and define

$$
K_{y}=\left\{\alpha: y_{\alpha} \neq 0\right\} \backslash K .
$$

Choose $x \in \Sigma_{K}$ so that $x_{\alpha}=y_{\alpha}$ if $\alpha \in K$. It follows that $y \in U_{\beta}(x)$ for $\beta$ sufficiently large, since $K_{y}$ is countable and since, whenever $\left(U_{\delta}(x)\right)_{\alpha} \neq J_{\alpha}$ and $\alpha \in K_{y}$, one has $\alpha \in K_{\beta}(x)$ for all $\beta>\delta$.

Second, it is now possible to prove that $\mathcal{H C}$ is the required refinement of $\mathcal{u}$. Let $H_{i} \in \mathcal{H}, i=1,2, \cdots$, let $x_{0} \in \bigcap_{1}^{\infty} H_{i}$ and let $y_{i} \in H_{i}$. Assume that $s_{0}$ is the smallest integer with the property that $H_{i_{0}} \in \mathcal{G}_{s_{0}}$ for some $H_{i_{0}} \in \mathcal{F C}$. Let $C$ be a countable subset of $A \backslash K$ such that $\left(y_{i}\right)_{\alpha}=0$ if $\alpha \in^{\prime} C$ and $i=1,2, \cdots$.

By the construction of $\mathcal{H C}, H_{i_{0}} \in \mathcal{F C}(z)$ for some $z \in \Sigma_{K}$. It will be shown that $\left\{y_{i}: i=1,2, \cdots\right\} \subset U_{\beta}(z)$ for $\beta$ large enough, where $U_{\beta}(z) \in \mathcal{H C}(z)$. Note first that for $\alpha \in K, U_{\beta}(z) \in \mathcal{H C}(z)$, and $U_{\delta}(z) \in \mathcal{H}(z)$, one has

$$
\left(U_{\beta}(z)\right)_{\alpha}=\left(U_{\delta}(z)\right)_{\alpha}=\left(H_{i_{0}}\right)_{\alpha} .
$$

Also, by the minimality of $s_{0}$ and the construction of $\mathcal{G}$,

$$
\left(y_{j}\right)_{\alpha_{i}}=\left(x_{\theta}\right)_{\alpha_{i}}=\left(y_{i_{0}}\right)_{\alpha_{i}}, \quad \text { if } i \leqq s_{0}, \quad \text { for all } j .
$$


Hence, for $\beta$ sufficiently large and $U_{\beta}(z) \in \mathcal{H C}(z)$, each $y_{i} \in U_{\beta}(z)$ because $C$ is countable and because, whenever $\left(U_{\delta}(z)\right)_{\alpha} \neq J_{\alpha}$ and $\alpha \in C$, one has $\alpha \in K_{\beta}(z)$ for $\beta>\delta$.

4. Completion of the proof of Theorem 1. It is sufficient to prove that a closed subspace of $\Sigma$ is not $\aleph_{0}$-fully normal. The space $F_{0}$ will be chosen for our purposes. Recall that $F_{0}$ is the set of $x \in \Sigma$ such that, for any integer $n \neq 0, x_{\alpha}=n$ for at most one $\alpha \in A$. Obviously $F_{0} \subset \Sigma$, and it is also a closed subspace, as was observed in [4].

Not only will it be possible to prove the announced result, but also the following stronger statement.

Lemma 2. The space $F_{0}$ is not 2-fully normal.

Proof. For the cover $w$ defined in the first paragraph of $\$ 3$, let $v$ be the trace of $W$ on $F_{0}$. Suppose $S$ is a refinement of $U$ by basic open sets such that $S_{1} \in \mathcal{S}, S_{2} \in \mathcal{S}$ and $S_{1} \cap S_{2} \neq \varnothing$ imply $S_{1} \cup S_{2} \subset V$ for some $V \in \mathcal{V}$. Let $x_{1} \in F_{0}$ be a point such that, for some nonempty, finite set $B_{1} \subset A,\left(x_{1}\right)_{\alpha}=0$ if $\alpha \in A \backslash B_{1}$ and $\left(x_{1}\right)_{\alpha} \neq 0$ if $\alpha \in B_{1}$.

Now I assert: There is a finite subset $B_{2}, B_{1} \subset B_{2} \subset A$, and a point $x_{2} \in F_{0}$ such that $x_{2}$ has the same relation to $B_{2}$ as $x_{1}$ does to $B_{1},\left(x_{2}\right)_{\alpha}=\left(x_{1}\right)_{\alpha}$ if $\alpha \in B_{1}$, and finally $x_{2} \in S \in S$ implies that $\left(x_{2}\right)_{\alpha}=S_{\alpha}$ for some $\alpha \in B_{2} \backslash B_{1}$.

To prove the assertion, let $S_{1} \in S$ be a set which contains $x_{1}$. Let

$$
B_{2}=\left\{\alpha \in A:\left(S_{1}\right)_{\alpha}=\left(x_{1}\right)_{\alpha}\right\} \cup B_{1},
$$

and choose any point $x_{2} \in F_{0}$ with the required relation to $B_{2}$ and $x_{1}$. It is easy to see that $B_{2}$ properly contains $B_{1}$, since $S$ is a refinement of $V$. Suppose $x_{2} \in S_{2} \in \delta$. Note that, since $S_{2}$ is basic, $\left(S_{2}\right)_{\alpha} \neq\left(x_{2}\right)_{\alpha}$ implies $\left(S_{2}\right)_{\alpha}=J_{\alpha}$ for $\alpha \in B_{2} \backslash B_{1}$. Hence if $S_{2}$ does not have the required property, $x_{1} \in S_{2}$. However, in this case $x_{1} \in S_{1} \cap S_{2}$ but no $V \in \mathcal{V}$ contains $S_{1} \cup S_{2}$, since $\left(S_{1}\right)_{\alpha}=0$ implies $\left(S_{2}\right)_{\alpha}=J_{\alpha}$. This proves the assertion.

It is clear that by replacing $x_{1}$ with $x_{2}$ and $B_{1}$ with $B_{2}$ one may choose $x_{3}$ and $B_{3}$ in the above manner, and so on. Now the sequence $\left\{x_{i}\right\}$ which is so chosen approaches some limit $x_{0} \in F_{0}$, since $\left(x_{i}\right)_{\alpha}=\left(x_{i+1}\right)_{\alpha}$ for all $\alpha \in \in^{\prime} B_{i+1} \backslash B_{i}$, and since $F_{0}$ is closed in $\Sigma$. Suppose $x_{0} \in S \in S$; then $x_{i} \in S$ for all $i$ sufficiently large. By the properties of $\left\{x_{i}\right\}$ and $\left\{B_{i}\right\}, x_{i+1} \in S$ implies that $S_{\alpha}=\left(x_{i+1}\right)_{\alpha}$ for some $\alpha \in B_{i+1} \backslash B_{i}$. It follows that $S$ is restricted simultaneously on infinitely many coordinates. Hence $S$ is not open, which is a contradiction.

CoRollaRy. $F_{0}$ is almost $\aleph_{0}$-fully normal and realcompact, but not 2-fully normal. (See [2] for the term realcompact.)

Proof. One may conclude that $F_{0}$ is realcompact from the results in [2].

Note that this corollary improves [2, Theorem 4]. Also, the proof of the corollary is more direct. 
5. Finite $m$. The case where $m$ is finite raises several problems, as Mansfield has observed. None of these are settled by the methods of this paper except the question concerning equivalence of $\mathfrak{m}$-full normality and almost $\mathfrak{m}$-full normality, $\mathfrak{m}$ finite. It is possible to prove that these properties are not equivalent for finite $m$ by a much simpler argument than that of $\S 3$, if one utilizes the theorems of [2], Lemma 2 of $\$ 4$ of this paper, and the following lemma. (Note that it is an open question whether almost 2-full normality implies almost $m$-full normality, $\mathfrak{m}$ finite, although the corresponding question concerning $\mathfrak{m}$-full normality has been answered affirmatively by A. J. Goldman. See [3].)

Lemma 3. Suppose $\mathfrak{m}$ is finite and $X^{2^{\mathfrak{m}}}$ is almost 2-fully normal. Then $X$ is almost $(\mathfrak{m}+1)$-fully normal.

Before proving Lemma 3 , note that it does imply that $\Sigma$ is almost $\mathfrak{m}$-fully normal for all finite $m$. In fact, it was proved in [2] that the collection of all the neighborhoods of the diagonal is a uniformity for $\Sigma^{\cdot}$ as long as $s \leqq \aleph_{0}$. Cohen discovered in [1] (see [3] for a proof) that the latter property is equivalent to almost 2 -full normality. Hence $\Sigma$ is almost $\mathfrak{m}$-fully normal for all finite $\mathfrak{m}$, but not $\mathfrak{m}$-fully normal because of the results of $\S 4$.

Proof of Lemma 3. The lemma is obvious when $\mathfrak{m}=1$, since $X$ is homeomorphic to a closed subspace of $X^{2}$, so suppose that it is true for $\mathfrak{m}-1$, and suppose $X^{2^{\mathfrak{m}}}=\left(X^{2}\right)^{2^{\mathfrak{m}-1}}$ is almost 2 -fully normal. Then $X^{2}$ is almost $\mathfrak{m}$-fully normal. Let $\mathcal{u}$ be an open cover of $X$. Define

$$
\mathcal{u}^{2}=\left\{U^{2}: U \in \mathcal{u}\right\} \text {. }
$$

There is a closed neighborhood $N$ of $\Delta$ in $X^{2}$ such that $N \subset \cup \mathcal{u}^{2}$. ( $\Delta$ is the diagonal in $X^{2}$. Hence $\Delta$ is closed in $X^{2}$, and the last statement follows from that fact that $X^{2}$ is normal.)

Since $X^{2}$ (and hence $N$ ) is almost m-fully normal, one may choose a refinement $V$ of the cover of $N$ by the trace of $\mathcal{u}^{2}$ on $N$ so that $U$ has these properties: If $V_{1} \cap \cdots \cap V_{\mathfrak{m}} \neq \varnothing, V_{i} \in \mathcal{V}$, and $\left\{p_{1} \cdots p_{\mathfrak{m}}\right\} \subset \cup_{1}^{\mathfrak{m}} V_{i}$, then there is a $U^{2} \in \mathcal{u}^{2}$ such that $\left\{p_{1} \cdots p_{\mathfrak{m}}\right\} \subset U^{2}$. For each point $x \in X$, let $T_{x}$ be an open neighborhood of $x$ such that $T_{x}^{2} \subset V$ for some $V \in V$. Since $X^{2}$ (and hence $X$ ) is almost 2-fully normal, there exists a refinement $\mathcal{H C}$ of $\left\{T_{x}: x \in X\right\}$ such that $H_{1} \cap H_{2} \neq \varnothing$ and $h_{i} \in H_{i} \in J C$ for $i=1$ and 2 implies that $\left\{h_{1}, h_{2}\right\} \subset T_{x}$ for some $x \in X$.

To show that $\mathcal{H}$ is the refinement that is required, let $\left\{x_{0} \cdots x_{m}\right\}$ be $\mathfrak{m}+1$ points in $\bigcup_{0}^{\mathfrak{m}} H_{i}$, where $H_{i} \in \mathcal{H C}$ and $\bigcap_{0}^{\mathfrak{m}} H_{i} \neq \varnothing$. By the relation of $\mathfrak{F C}$ to $\left\{T_{x}: x \in X\right\}$, there is for each $i, 0 \leqq i \leqq \mathfrak{m}$, a $T_{y_{i}}$ such that $\left\{x_{0}, x_{i}\right\} \subset T_{y_{i}}^{2}$. Notice that $\left(x_{0}, x_{i}\right) \in T_{y_{i}}^{2}$ and $\left(x_{0}, x_{0}\right) \in T_{y_{i}}^{2}$, for each $i$. Hence $\bigcap_{i}^{\mathfrak{m}} T_{y_{i}}^{2} \neq \varnothing$. Therefore $U_{1}^{m}\left\{\left(x_{0}, x_{i}\right)\right\} \subset U^{2}$ for some $U^{2} \in \mathcal{u}^{2}$, since each $T_{i}^{2} \subset V_{i}$ for some $V_{i} \in \mathcal{V}$. From this one may conclude that $\left\{x_{0} \cdots x_{m}\right\} \subset U$. 
REMARK. I could not find a way to apply this simpler approach to Theorem 1.

\section{REFERENCES}

1. H. J. Cohen, Sur un probleme de M. Dieudonne, C. R. Acad. Sci. Paris vol. 234 (1952) pp. 290-292.

2. H. H. Corson, Normality in subsets of product spaces, Amer. J. Math. vol. 81 (1959) pp. 785-796.

3. M. J. Mansfield, Some generalizations of full normality, Trans. Amer. Math. Soc. vol. 86 (1957) pp. 489-505.

4. A. H. Stone, Paracompactness and product spaces, Bull. Amer. Math. Soc. vol. 54 (1948) pp. 977-982.

UNIVERSITY OF WASHINGTON,

Seattle, Washington 